\title{
Universal Large-order asymptotic behavior of the Strong-coupling and High-Temperature series expansions
}

\author{
Abouzeid M. Shalaby* \\ Department of Mathematics, Statistics, and Physics, \\ Qatar University, Al Tarfa, Doha 2713, Qatar
}

\begin{abstract}
For theories that exhibit second order phase transition, we conjecture that the large-order asymptotic behavior of the strong-coupling ( High-Temperature) series expansion takes the form $\sigma^{n} n^{b}$ where $b$ is a universal parameter. The associated critical exponent is then given by $b+1$. The series itself can be approximated by the hypergeometric approximants ${ }_{p} F_{p-1}$ which can mimic the same large-order behavior of the given series. Near the tip of the branch cut, the hypergeometric function ${ }_{p} F_{p-1}$ has a power-law behavior from which the critical exponent and critical coupling can be extracted. The conjecture has been tested in this work for the perturbation series of the ground state energy of the Yang-Lee model as a strong-coupling form of the $\mathcal{P} \mathcal{T}$-symmetric $i \phi^{3}$ theory and the High-Temperature expansion within the Ising model. From the known $b$ parameter for the Yang-Lee model, we obtained the exact critical exponents which reflects the universality of $b$. Very accurate prediction for $b$ has been obtained from the many orders available for the High-Temperature series expansion of the Ising model which in turn predicts accurate critical exponent. Apart from critical exponents, the hypergeometric approximants for the Yang-Lee model show almost exact predictions for the ground state energy from low orders of perturbation series as input.

PACS numbers: 02.30.Lt,64.70.Tg,11.10.Kk
\end{abstract}

Keywords: Universality, $\mathcal{P} \mathcal{T}$-symmetry, Hypergeometric Resummation, Strong-Coupling expansion

*amshalab@qu.edu.qa 


\section{INTRODUCTION}

Quantum filed theory represents one of the most successful tools to study critical phenomena in physics. The point is that one can have different models behave similarly near the critical point. In this case we say that these models are in the same class of university where critical exponents (for instance) are the same for the whole class. The Ising model from magnetism and the $\phi^{4}$ scalar field theory reflect that belief as both are well known to lie in the same class of universality. Near the critical point, however, perturbations (weak-coupling) always fail to give reliable results. The reason behind this is that the effective coupling blows up and turns the theory highly non-perturbative for which one has to employ rigorous nonperturbative techniques to be able to extract reliable results. From a mathematical point of view, the weak-coupling expansion diverges because one expands around a point which represents an essential singularity of the theory [1-3]. Accordingly, one can expect that the expansion around another point in the coupling space might lead to a different behavior of the perturbation series. This is what strong-coupling expansion in field theory [3-5] and the equivalent high-temperature (HT) expansion in statistical systems $[6,7]$ are expected to do. A note to be mentioned is that the mathematical structure of the strong coupling expansion in lattice field theory is equivalent to the high-temperature (HT) expansion in condensed matter physics [8-11]. Also, lattice spacing serves as a suitable regularization of the theory under consideration. Being expanded around a point of no essential singularity, the strong-coupling expansion is known to have a finite radius of convergence [12] similar to the ${ }_{p} F_{p-1}$ hypergeometric approximants while the weak-coupling expansion for the $\phi^{4}$ or $i \phi^{3}$ (for instance) field theories has a zero radius of convergence manifested by the $n$ ! growth factor in the large-order asymptotic behavior of the series.

Near second order phase transition, a physical quantity $Q(T)$, where $T$ is the temperature, has a power-law behavior of the from $Q(T) \propto{ }_{1} F_{0}\left(\psi ; ; \frac{T}{T_{c}}\right)=\left(1-\frac{T}{T_{c}}\right)^{-\psi}$ where $\psi$ is called a critical exponent while $T_{c}$ is the critical temperature. In fact, all the hypergeometric functions ${ }_{k+1} F_{k}\left(a_{1}, a_{2}, \ldots a_{k+1} ; b_{1}, b_{2}, \ldots b_{k} ; \frac{T}{T_{c}}\right)$ have such power-law behavior near the tip of the branch cut which mimics the critical point. The hypergeometric series ${ }_{k+1} F_{k}\left(a_{1}, a_{2}, \ldots a_{k+1} ; b_{1}, b_{2}, \ldots b_{k} ; \frac{T}{T_{c}}\right)$ has a finite radius of convergence as its large-order 
asymptotic behavior looks like $\left(-\frac{1}{T_{c}}\right)^{n} n^{b}$ where [13]:

$$
b=\psi-1=\sum_{i=1}^{k+1} a_{i}-\sum_{j=1}^{k} b_{j}
$$

So it is clear that the large-order parameter $b$ of a series with finite radius of convergence totally defines the critical exponent and thus is expected to be universal, the same way critical exponents do.

The power-law behavior characterizing the second order phase transition is itself the member ${ }_{1} F_{0}$ of the set of hypergeometric approximants ${ }_{k+1} F_{k}\left(a_{1}, a_{2}, \ldots a_{k+1} ; b_{1}, b_{2}, \ldots b_{k} ; \frac{T}{T_{c}}\right)$. Accordingly, the critical exponent can be shown easily to be related to the large-order asymptotic behavior of the expansion of the power- law formula. Let us rewrite the critical behavior in a another but equivalent form:

$$
\begin{aligned}
Q(T) & \propto\left(T-T_{c}\right)^{-\psi} \\
& =T^{-\psi}\left(1-\frac{T_{c}}{T}\right)^{-\psi} .
\end{aligned}
$$

For non-trivial transition $\left(T_{c} \neq 0\right)$, we can have a high temperature expansion of $Q(T)$ which again has a finite-radius of convergence with the parameter $b$ again is given by $b=\psi-1$. The relation $b=\psi-1$, although proved by considering hypergeometric approximants, is in fact general and is a manifestation of the theorem of Darboux which implies that late terms of the expansion of a power-law form and that of a Taylor series of the given quantity are of the same form [14] provided that the series has a finite radius of convergence.

Our conjecture for the existence of an expansion with a universal $b$ parameter motivates for the study of second-order phase transition within the strong-coupling expansion in quantum field theory. The fact that the strong-coupling (HT) expansion has a finite radius of convergence will be stressed in sec.II. Our idea for the preference to study critical phenomena within strong-coupling expansion is very important as there are quasi-classical techniques that are supposed to obtain the exact large-order asymptotic behavior of that expansion. Thus, for the sake of getting the first exact critical exponent in three dimensions, it is worth it to make the needed effort to study the strong-coupling expansion in field theory. Such kind of studies can relate critical exponents to the asymptotic large-order parameter $b$ which we expect to be universal. 
In this paper, we shall stress the Yang-Lee model as a strong-coupling expansion of a field theory in $0+1$ dimensions. For that model, the asymptotic large-order behavior is known and and thus can be used to show that the parameter $b$ is universal. Taking into account that the HT-expansion in condensed matter and strong-coupling from lattice field theory are two sides of the same coin [9-11, 15-19] and that the high-temperature (strong-coupling) expansion is known up to a relatively high order, we shall stress that expansion for both SQ and SC lattices for the Ising model and show again that the parameter $b$ is universal.

The rest of this paper is organized as follows. In sec.II, we highlight the fact that the strong-coupling (HT) expansion has a finite radius of divergence. In sec III, the weakcoupling, strong-coupling and large-order parametrization of the hypergeometric approximants is stressed. In sec.IV, we apply the hypergeometric approximation for the series of the ground state-energy of the Yang-Lee model. In this section, all critical exponents are obtained exactly from knowing the $b$ parameter in the large-order behavior. In sec.V, the HT-expansion of the susceptibility within the SC lattice of the Ising-model is investigated while the SQ case is investigated in sec.VI. Using the last highest orders (large $n$ ) of the known 25 orders of the associated perturbation series, we were able to obtain very accurate approximation for the parameter $b$ which in turn shows its universality via comparison with the well known results for the $\gamma$ exponent. Summary and conclusions will follow in sec.VII.

\section{LARGE-ORDER ASYMPTOTIC BEHAVIOR FROM THE STRONG-COUPLING EXPANSION IN QUANTUM FIELD THEORY}

There is a one-to-one correspondence between the $n$ ! growth factor in the large-order asymptotic behavior of the week-coupling expansion and the essential singularity existing at zero coupling $[2,3]$. For the $\phi^{4}$ scalar field theory, for instance, the large-order asymptotic behavior for the weak-coupling expansion takes the form $n ! \sigma^{n} n^{b}$. In Refs.[13, 20, 21], we showed that a series of such behavior ( it has a zero-radius of convergence) can be fitted by the hypergeometric approximants ${ }_{p+1} F_{p-1}\left(a_{1}, \ldots a_{p+1} ; b_{1} \ldots b_{p-1} ; \sigma z\right)$. These hypergeometric approximants can be analytically continued to non-zero $z$ values via their representation in terms of the Meijer G function. On the other hand, the strong-coupling (High-Temperature)

expansion is well known to have a finite radius of convergence [3, 12] and thus the asymptotic large-order behavior is taking the form $\sigma^{n} n^{b}$ without an $n$ ! growth factor found in the 
week-coupling expansion. In Ref.[13], we showed that such type of series ( with finite radius of convergence) can be approximated by a different type of hypergeometric approximants ( $\left.{ }_{p+1} F_{p}\left(a_{1}, \ldots a_{p+1} ; b_{1} \ldots b_{p} ; \sigma z\right)\right)$. These approximants can produce the same large-order asymptotic behavior with their parameters are constrained as :

$$
b+1=\sum_{i=1}^{p+1} a_{i}-\sum_{j=1}^{p} b_{j}
$$

Near the branch cut, the approximants ${ }_{p+1} F_{p}\left(a_{1}, \ldots a_{p+1} ; b_{1} \ldots b_{p} ; \sigma z\right)$ have a power-law behavior of the form:

$$
{ }_{p+1} F_{p}\left(a_{1}, \ldots a_{p+1} ; b_{1} \ldots b_{p} ; \sigma z\right) \sim(1-\sigma z)^{-\psi}
$$

where $\psi=b+1$. Accordingly, knowing the large order parameter $b$ of the strong-coupling expansion will lead to the exact determination of the critical exponent while knowing the parameter $\sigma$ will determine the critical coupling.

In literature, there exist quasi-classical techniques (out of the scope of this work) for the exact determination of the large-order parameters $b$ and $\sigma$ [3, 22]. However, up to the best of our knowledge this issue has not been stressed rigorously for the strong-coupling expansion in field theory in other than one dimensional cases (quantum mechanics). Fortunately, strong-coupling (High-Temperature) expansions for many models are listed in literature up to high orders $[6,7,15,23,24]$ which means that one can extract approximate values of the parameters $b$ and $\sigma$. So at least approximately, one can test the validity of our conjecture about the universality of $b$ by extracting this parameter from the relatively high number of terms available in literature. Confirming the universality of $b$ might open the door for the first determination of exact critical exponents from the exact determination of $b$ for the strong-coupling (HT) expansion.

Before we try to test our conjecture, let us first highlight the fact that the strong-coupling (High-temperature) expansion possesses a finite radius of convergence and thus at large $n$, the the $n^{\text {th }}$ coefficient behaves like $\sigma^{n} n^{b}$. To do that, in the following, we list different Hamiltonian models for which the strong coupling expansions can be shown to have a finite radius of convergence: 


\section{II.1. Large-order asymptotic behavior of the strong-coupling expansion for anharmonic oscillators}

The Hamiltonian of the anharmonic oscillators is given by

$$
H_{s}=p^{2}+x^{2}+\beta x^{2 m}
$$

Here $\beta$ is the coupling constant (should not be confused with inverse temperature $\beta$ ). The strong-coupling of this Hamiltonian has been stressed in Ref.[25] and the ground state energy has been shown to have an expansion of the form:

$$
E_{0}=\beta^{\frac{1}{m+1}} \sum_{n=0}^{\infty} h_{n} \beta^{\frac{-2 n}{m+1}},
$$

where for the limit $n \rightarrow \infty$ we have the asymptotic form:

$$
h_{n} \sim c n^{-\frac{3}{2}} \sigma^{n}\left(1+O\left(\frac{1}{n}\right)\right) .
$$

The ratio test can tell us clearly that the strong-coupling series above has a finite radius of convergence with the parameter $b$ having its exact value of $-3 / 2$. One can realize that $b=\frac{-3}{2}$ for the different Hamiltonians (different $m$ values) in the set. It is well known that at the Ising limit $(\beta \rightarrow \infty)$, physical quantities behave similarly for different interaction Hamiltonian [16]. As long as $b$ is same for different $m$, one might conclude that it is a universal quantity. Also one can conclude that $b=\frac{-3}{2}$ for the $\mathcal{P} \mathcal{T}$-symmetric $i x^{3}$ Hamiltonian as well. In the next section we shall see that this value determines the known exact critical exponent for that model.

Since $b$ defines the associated critical exponent and so far is not known exactly for field theories in higher dimensions, we shall try to obtain an approximation for $b$ from the strongcoupling expansion of the $\mathcal{P} \mathcal{T}$-symmetric $i x^{3}$ model and compare it with the exact result. After that we can extend the same strategy for the strong-coupling expansion in field theory to get an approximate value for $b$ and thus test its universality.

For large $n$, the ratio $R_{n}=h_{n} / h_{n-1}$ can be approximated as :

$$
\begin{aligned}
R_{n} & =\frac{c n^{b} \sigma^{n}}{c(n-1)^{b} \sigma^{n-1}}=\sigma\left(1-\frac{1}{n}\right)^{b} \\
& \simeq \sigma-b \sigma\left(\frac{1}{n}\right)
\end{aligned}
$$


Accordingly, $R_{n}$ for series with finite radius of convergence can be fitted with a straight line when plotted versus $\frac{1}{n}$ (for large $n$ ). The first twenty coefficients of the series in Eq.(13) in Sec.IV have been obtained in Ref.[26] ( Eq.(92) there). In Fig.1, we plotted $R_{n}$ versus $\frac{1}{n}$ where the data can be fitted by the equation:

$$
R_{n}=1.1937\left(\frac{1}{n}\right)-0.7429 .
$$

From this equation one can conclude approximate values for the large order parameters as $b=-1.6068$ compared to its exact value $b=\frac{-3}{2}$ and $\sigma=0.7429$. In sec.IV, we shall see that fitting the series using the hypergeometric approximants ${ }_{p+1} F_{p}\left(a_{1}, \ldots a_{p+1} ; b_{1} \ldots . b_{p} ; \sigma z\right)$ can give better values for $\sigma$ which determines the critical coupling. The plot in Fig.1 is thus

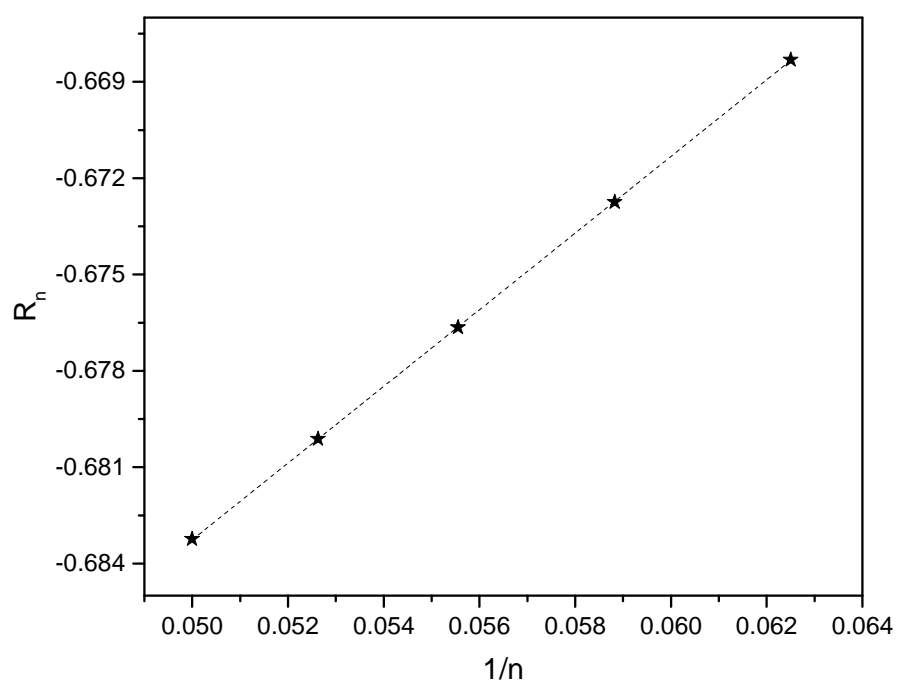

FIG. 1. In this figure, we plot the coefficients ratio $R_{n}$ for the strong-coupling expansion of the vacuum energy of the $\mathcal{P} \mathcal{T}$-symmetric ix $x^{3}$ model (obtained in Ref.[26]) at large orders. The data (stars) has a straight line fit (dashed) of the form $R_{n}=1.1937\left(\frac{1}{n}\right)-0.7429$ which predicts the values $\sigma=-0.7429$ and $b=-1.6068$.

showing that one can get approximate values for the parameters $b$ and $\sigma$ from the last few orders in the strong-coupling expansion. 


\section{II.2. Strong-coupling expansion for the $g \phi_{1+1}^{4}$ vacuum energy}

In Ref.[23], strong-coupling series (lattice) for the vacuum energy of the $g \phi_{1+1}^{4}$ scalar filed theory has been obtained up to $11^{\text {th }}$ order in $y=g^{-\frac{2}{3}}$ as:

$$
\begin{aligned}
E & =g^{\frac{1}{3}} e_{g} \\
e_{g} & \simeq 0.66798625915577710827096201688+0.43100635014259473006095738275 \lambda \\
& +\ldots \ldots \ldots \ldots-0.0087493465269972 \lambda^{8}+0.007096747591805 \lambda^{9}-0.005871428 \lambda^{10} \\
& +0.0049362 \lambda^{11} .
\end{aligned}
$$

The ratio test can confirm the convergence of the series for $e_{g}$. We plot $R_{n}$ versus $\frac{1}{n}$ in Fig.2 where the fitting gives $\sigma=0.9726$ and $b=-1.4933$. These results can be checked by the radius of convergence $\frac{1}{\sigma}=1.0282$ which is very close to the findings in Ref.[23]. Of course the hypergeometric approximants ${ }_{p+1} F_{p}\left(a_{1}, \ldots a_{p+1} ; b_{1} \ldots b_{p} ; \sigma \lambda\right)$ can be parametrized to fit the given series with better approximation for the values of $\sigma$ and $b$.

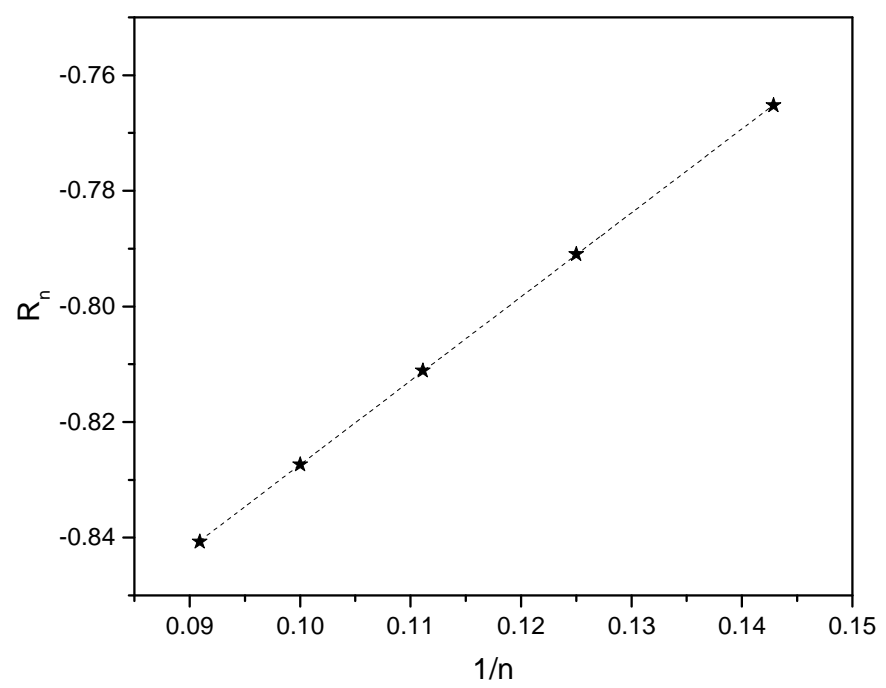

FIG. 2. In this figure, we plot the ratio $R_{n}$ for the strong-coupling expansion (lattice) of the vacuum energy for the $\phi^{4}{ }_{1+1}$ scalar field theory at large $n$. The data has a straight line fit of the form $R_{n}=1.4524\left(\frac{1}{n}\right)-0.97261$ which predicts the values $\sigma=0.9726$ and $b=-1.4933$. 


\section{II.3. High-Temperature (Strong-coupling) expansion for the $O(2)$-symmetric $\phi_{2+1}^{4}$ model}

In Ref.[24], the High-Temperature (strong-coupling) expansion for the second moment of the two-point function for the $O(2)$-symmetric $\phi_{2+1}^{4}$ scalar field theory is listed up to $\beta^{20}$, where $\beta$ is the inverse temperature (column 2 in TABLE XVII there). In Fig.3, we generated the plot for $R_{n}$ versus $1 / n$ and extracted the values $b=1.7485$ while $\sigma=1.9575$.

The critical inverse temperature $\beta_{c}=\frac{1}{\sigma}=0.51086$ compared to the result $\beta_{c}=0.5099049$ in Ref.[24]. Again, the hypergeometric approximants are expected to give better predictions but we will not stress it here as our aim from this section is to highlight the fact known from literature that the strong-coupling expansion has a finite radius of convergence and thus having a large order asymptotic behavior similar to that of the hypergeometric series ${ }_{p+1} F_{p}\left(a_{1}, \ldots a_{p+1} ; b_{1} \ldots b_{p} ; \sigma \beta\right)$.

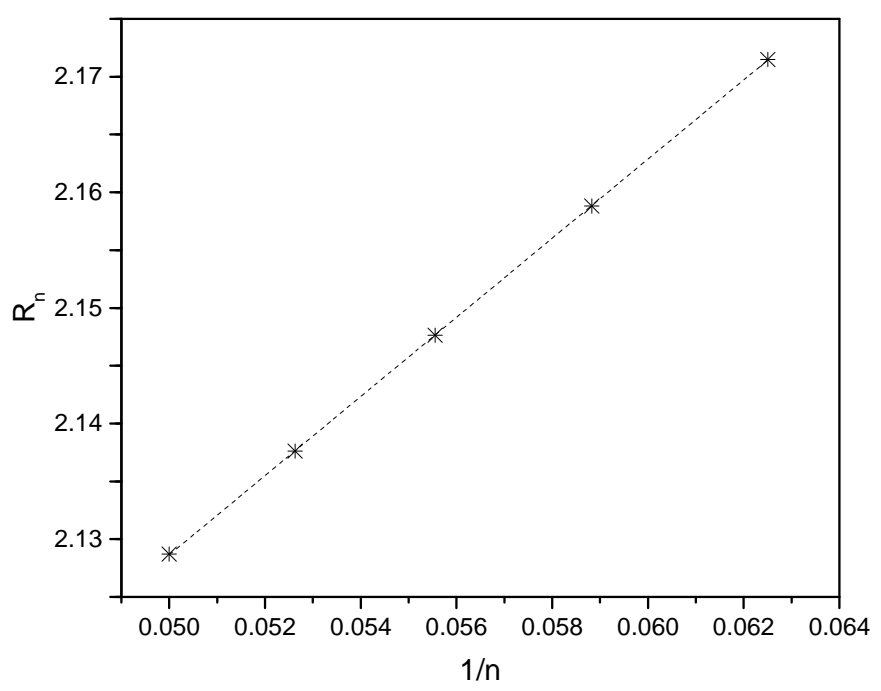

FIG. 3. The plot of $R_{n}$ for the High-Temperature (strong-coupling) expansion (lattice) of the second moment correlation function of the of the three-dimensional $\phi^{4}$ field theory with $O(2)$ symmetry. The data can be fitted as $R_{n}=3.4227\left(\frac{1}{n}\right)+1.9575$ which predicts the values $\sigma=1.9575$ and $b=-3.4227$. 


\section{WEAK COUPLING, STRONG-COUPLING AND LARGE-ORDER PARAMETRIZATION OF THE HYPERGEOMETRIC RESUMMATION}

Famous non-perturbative tools that are always used in literature to study critical phenomena in physics are Borel, Borel-Padé and Borel with conformal mappings resummation algorithms [22, 27-33]. In applying these algorithms one may face slow convergence and most of the calculations are achieved using numerical steps. Recently, the simple but accurate hypergeometric resummation algorithm has been introduced which is of closed form [34]. In Ref.[35], we showed that one can employ the strong-coupling data to determine all the numerator parameters in the hypergeometric ${ }_{p} F_{p-1}$ approximants. In another work [13, 20], we showed that the relation between $p$ (number of numerator parameters) and $q$ (number of denominator parameters) is constrained by the large-order behavior of the given perturbation series. Once we determined the difference $p-q$ from the large order behavior, one can employ the large order parameters to accelerate the convergence of the hypergeometric resummation.

Based on the large order-behavior of a given perturbation series, one can categorize the divergent series into different classes, where each class can be resummed by a Hypergeometric function with expansion that reflects the needed growth factor [13, 20]. To clarify this point more, consider a divergent series for a physical quantity $Q(z)=\sum_{n=0}^{n} c_{n} z^{n}$ that has the following large-order behavior:

$$
c_{n} \sim \alpha((p-q-1) n) !(-\sigma)^{n} n^{b}\left(1+O\left(\frac{1}{n}\right)\right), \quad n \rightarrow \infty .
$$

Such types of asymptotic large-order behavior suggest the following approximants:

1. For a series with finite radius of convergence, we have $(p-q-1)=0$. The suitable hypergeometric approximant is then

$$
\begin{aligned}
Q(z) & \approx c_{0} F_{p-1}\left(a_{1}, \ldots a_{p} ; b_{1} \ldots b_{p-1} ;-\sigma z\right), \text { where } \\
b & =\sum_{i=1}^{p} a_{i}-\sum_{i=1}^{p-1} b_{i}-1 .
\end{aligned}
$$

2. For a divergent series with zero radius of convergence and $n$ ! growth factor $(p-q-1=$ 
1), the suitable approximant is then

$$
\begin{aligned}
Q(z) & \approx c_{0 p} F_{p-2}\left(a_{1}, \ldots a_{p} ; b_{1} \ldots b_{p-2} ;-\sigma z\right), \\
b & =\sum_{i=1}^{p} a_{i}-\sum_{i=1}^{p-2} b_{i}-2 .
\end{aligned}
$$

3. For a divergent series with zero-radius of convergence but $(2 n)$ ! growth factor $(p-q-$ $1=2$ ) ( the ground state energy of the sixtic oscillator for instance) then the suitable approximant is

$$
Q(z) \approx c_{0} F_{p-3}\left(a_{1}, \ldots a_{p} ; b_{1} \ldots b_{p-3} ;-\sigma z\right)
$$

and so on. Of course for $p \geq q+2$, the series ${ }_{p} F_{q}$ is divergent and has a zero-radius of convergence but analytic continuation to non-zero $z$ values can be offered by a MellinBarnes integral representation of ${ }_{p} F_{q}$ or equivalently in terms of the Meijer-G function $[13,20]$.

We call the above algorithm the Hypergeometric- Meijer resummation [13, 20]. Note that, in this algorithm once you select the suitable Hypergeometric approximant based on the growth factor in the large order behavior, it can accommodate all weak-coupling, strong coupling and large-order data associated with the given perturbation series. In fact, in Ref.[36], Mera et.al used Borel-hypergeometric algorithm with Borel functions of the form $c_{0} F_{p-1}\left(a_{1}, \ldots a_{p} ; b_{1} \ldots . b_{p-1} ; . \sigma z\right)$. That algorithm results in a Meijer-G function resummation approximant that employs low order perturbation data as input. In our technique, we do not use any Borel or Padé methods but instead we start from large order behavior and select the appropriate hypergeometric approximant. In case $p-q$ is greater than one, we use the Meijer-G function representation of the Hypergeometric function [37] where

$$
{ }_{p} F_{q}\left(a_{1}, \ldots a_{p} ; b_{1} \ldots . . b_{q} ; z\right)=\frac{\prod_{k=1}^{q} \Gamma\left(b_{k}\right)}{\prod_{k=1}^{p} \Gamma\left(a_{k}\right)} G_{p, q+1}^{1, p}\left(\begin{array}{c}
1-a_{1}, \ldots, 1-a_{p} \\
0,1-b_{1}, \ldots, 1-b_{q}
\end{array} \mid z\right) .
$$

The Meijer $\mathrm{G}$ function on the right hand side of this equation has the integral representation of the form:[37]:

$$
G_{p, q}^{m, n}\left(\begin{array}{l}
c_{1}, \ldots, c_{p} \\
d_{1}, \ldots, d_{q}
\end{array} \mid z\right)=\frac{1}{2 \pi i} \int_{C} \frac{\prod_{k=1}^{n} \Gamma\left(s-c_{k}+1\right) \prod_{k=1}^{m} \Gamma\left(d_{k}-s\right)}{\prod_{k=n+1}^{p} \Gamma\left(-s+c_{k}\right) \prod_{k=m+1}^{q} \Gamma\left(s-d_{k}+1\right)} z^{s} d s .
$$


By selecting the contour $C$ to run from from $-i \infty$ to $+i \infty$ [37], the integral above converges for $p+q<2(m+n)$. It is then clear that for $p=q+1$ where the hypergeometric series has a finite radius of convergence, the condition for the convergence of the above integral is satisfied.

The algorithm has been shown to give accurate results for different divergent series like the ground state energy of anharmonic oscillator [20,35] and the critical exponents of the $O(N)$-symmetric model $[13,21]$. In this work, however, we will concentrate on resummation of strong-coupling (High-Temperature ) series expansion for systems that show up second order phase transition. Such type of series have a finite radius of convergence and thus hypergeometric approximants ${ }_{p} F_{q}$ with $p-q-1=0$ are relevant ones. Near the tip of the branch cut, the approximants posses a power-law behavior where the critical exponents are solely determined by the large-order parameter $b$ while $\sigma$ determines the critical coupling (or temperature).

\section{HYPERGEOMETRIC APPROXIMATION FOR THE STRONG-COUPLING EXPANSION OF THE YANG-LEE MODEL}

In 1952, Lee and Yang introduced a theory of phase transitions that is based on the zeros of the partition function in the complex plane of an external parameter like the external magnetic field $[38,39]$. At the continuum limit, the zeros of the partition function can touch the real axis which then represents a critical point called Yang-Lee edge singularity. For many years the zeros of partition function is considered as a theoretical issue but recently it has been exposed to experimental investigations (see Ref.[40] and references therein). In fact the zeros of the partition function are always existing for non-real external parameters and thus the theory near the zeros can turn to be non-Hermitian but $\mathcal{P} \mathcal{T}$-symmetric [41]. The link between critical behavior of the Ising model near edge singularity and $\mathcal{P} \mathcal{T}$-symmetric $i \phi^{3}$ theory was first introduced by Fisher who identified an effective action of the magnetization of the Ising model as a Landau-Ginzberg theory given by a $\mathcal{P} \mathcal{T}$-symmetric $i \phi^{3}$ theory [42]. We will study this model in $0+1$ space-time dimension and tackle the critical behavior associated to the edge singularity from the point of view of the dependance of the order parameter on the external magnetic field rather than investigating the zeros of partition function. 
Near the edge singularity, perturbative calculation within the Yang-Lee quantum field model can't account for the expected phase transition. In this model the Lagrangian density is given by:

$$
\mathcal{L}[\phi]=\frac{1}{2}(\partial \phi)^{2}-\frac{1}{2} m^{2} \phi^{2}(x)-\frac{i g}{3} \phi^{3}(x)+i J \phi(x) .
$$

We studied this model in Ref.[43] and showed that in $d=6-\epsilon$ dimensions, there exists a Gaussian fixed point where exact critical exponents are extracted from the one-loop effective potential. In the same reference we showed that for dimensions $d<6$, the effective coupling $\left(\frac{g}{M^{3-\frac{1}{2} d}}\right)$ blows up and the the theory has non-perturbative fixed point. In these cases, the one-loop effective potential would not be able to produce reliable results near the critical point. The worst case exists for $d=1$, where at the critical point $(M \rightarrow 0)$ the effective coupling blows up very fast. We used the effective potential to study this case but faraway from the critical region in Ref.[44]. This theory is $\mathcal{P} \mathcal{T}$-symmetric [45, 46] and the $\mathcal{P} \mathcal{T}$ symmetry is broken at the fixed point [47, 48]. At this point there exists a phase transition at which we showed ( for $d=6-\epsilon$ ) [43] that the fixed point is really representing a Yang-Lee edge singularity [38, 39, 42, 49-57].

The effective action of the magnetization of the Ising model has a Landau-Ginzburg representation at the continuum limit of the form [42]

$$
S=\int d x^{d}\left(\frac{1}{2}\left(\partial_{\mu} \phi\right)^{2}+i\left(h-h_{c}\right) \phi+i g \phi^{3}\right),
$$

which is equivalent to the Yang-Lee model above. The critical exponents associated with the Yang-Lee edge singularity have been listed in Ref.[42]. The study of that reference relied on considering the density of zeros of the partition function which has been shown to follow a power-law behavior near the edge singularity exactly the same manner the magnetization follows with respect to the external magnetic field.

The $\mathcal{P} \mathcal{T}$-symmetric Yang-Lee model in $0+1$ space-time dimension (quantum mechanics) has been studied in Ref.[26]. The Hamiltonian of that model in one dimension is given by:

$$
H_{g}=\frac{\pi^{2}}{2}+\frac{1}{2} m^{2} \phi^{2}+\frac{i \sqrt{g}}{6} \phi^{3} .
$$

The weak-coupling series expansion of the ground state energy of that model is divergent and thus resummation techniques are to be followed to get reliable results [26]. A strong coupling representation can be obtained using a scale and shift transformations [26, 35] that 
leads to the form:

$$
H_{g}=\sqrt[5]{g}\left(\frac{\pi^{2}}{2}+\frac{i \phi^{3}}{6}+\frac{1}{2} \frac{i m^{4}}{g^{\frac{4}{5}}} \phi\right)-\frac{m^{6}}{3 g} .
$$

This Hamiltonian can be rewritten as $H_{g}=g^{\frac{1}{5}} H_{J}-\frac{m^{6}}{3 g}$ where

$$
H_{J}=\frac{\pi^{2}}{2}+\frac{i \phi^{3}}{6}+\frac{1}{2} i J \phi
$$

with $J=\frac{i m^{4}}{g^{\frac{4}{5}}}$. The Hamiltonian $H_{J}$ has been studied also in Ref. [26] where the ODM method is used to resum the divergent series representing the ground state energy $E_{0}^{J}$ where

$$
\begin{aligned}
E_{0}^{J} & =\sum_{n=0}^{\infty} d_{n} J^{n}=.3725457904522070982506011+0.3675358055441936035304 J \\
& +0.1437877004150665158339 J^{2}+O\left(J^{3}\right) .
\end{aligned}
$$

As we explained in the introduction, near critical point physical quantities follow a powerlaw behavior. The power law behavior of the form $\left(J-J_{c}\right)^{\delta}$ has a series expansion with finite radius of convergence and a large-order asymptotic behavior like $\sigma^{n} n^{b}$, where $b=-\delta-1$ and $J_{c}=1 / \sigma$. According to the theorem of Darboux, the large order terms in an expansion originates from the singularity (closest to origin) of the expanded function [14]. Accordingly, one can expect that large-order form $\sigma^{n} n^{b}$ of the singular part (power-law) and the largeorder asymptotic behavior of the whole series (assumed to have a finite radius of convergence like the power-law form) have the same form. Accordingly, one concludes the direct relation between the parameter $b$ and the critical exponent. Note that the weak-coupling expansion has an essential singularity and thus Darboux theorem is not applicable for that case.

The most suitable hypergeometric approximant for a perturbation series is determined from the large order behavior of that series. In fact, for a class of interaction Hamiltonian $\beta x^{m}$, the large order behavior has been obtained in Ref.[25]. In fact, the large order behavior for the Hamiltonian $H_{J}$ can be concluded from that reference if we set $m=\frac{3}{2}$ in the Hamiltonian there where:

$$
H_{m}=p^{2}+x^{2}+\beta x^{2 m}
$$

The ground state energy of the rescaled Hamiltonian $\beta^{\frac{2}{5}} H_{m}$ has the expansion

$$
E_{0}^{m}=\sum_{n=0}^{\infty} h_{n} \beta^{\frac{-2 n}{m+1}}
$$


where for the limit $n \rightarrow \infty$ we have the asymptotic form:

$$
h_{n} \sim c n^{-\frac{3}{2}} \sigma^{n}\left(1+O\left(\frac{1}{n}\right)\right) .
$$

Note that the parameter $b=-\frac{3}{2}$ in the large order above does not depend on $m$ which reflects a kind of universality of the whole class and thus we can extend it to the case of the Yang-Lee model represented by the perturbation series in Eq.(13). As we expected, this large order behavior tells us that the strong-coupling series expansion has a finite radius of convergence. What is important in the above large-order behavior is that the hypergeometric function ${ }_{p} F_{p-1}\left(a_{1}, \ldots a_{p} ; b_{1} \ldots b_{p-1} ;-\sigma z\right)$ has the same form of large order behavior of its expansion. This can be shown by noting that:

$$
{ }_{p} F_{p-1}\left(a_{1}, \ldots \ldots a_{p} ; b_{1}, \ldots \ldots . . b_{q} ;-\sigma z\right)=\sum_{n=0}^{\infty} \frac{\frac{\Gamma\left(a_{1}+n\right)}{\Gamma\left(a_{1}\right)} \ldots . . \frac{\Gamma\left(a_{p}+n\right)}{\Gamma\left(a_{p}\right)}}{n ! \frac{\Gamma\left(b_{1}+n\right)}{\Gamma\left(b_{1}\right)} \ldots . \frac{\Gamma\left(b_{p-1}+n\right)}{\Gamma\left(b_{p-1}\right)}}(-\sigma z)^{n},
$$

and thus has a large order behavior of the form in Eq.(14) but with

$$
\frac{\frac{\Gamma\left(a_{1}+n\right)}{\Gamma\left(a_{1}\right)} \ldots \frac{\Gamma\left(a_{p}+n\right)}{\Gamma\left(a_{p}\right)}}{n ! \frac{\Gamma\left(b_{1}+n\right)}{\Gamma\left(b_{1}\right)} \ldots . \frac{\Gamma\left(b_{p-1}+n\right)}{\Gamma\left(b_{p-1}\right)}}(-\sigma)^{n} \sim \gamma(-\sigma)^{n} n^{b}\left(1+O\left(\frac{1}{n}\right)\right), \quad n \rightarrow \infty,
$$

where

$$
\sum_{i=1}^{p} a_{i}-\sum_{i=1}^{p-1} b_{i}-1=b
$$

and

$$
\gamma=\frac{\prod_{i=1}^{p-1} \Gamma\left(b_{i}\right)}{\prod_{i=1}^{p} \Gamma\left(a_{i}\right)}
$$

We can obtain the above relations easily using the asymptotic form of a ratio of two $\Gamma$ functions [58]:

$$
\frac{\Gamma(n+\alpha)}{\Gamma(n+\beta)}=n^{\alpha-\beta}\left(1+\frac{(\alpha-\beta)(-1+\alpha+\beta)}{n}+O\left(\frac{1}{n^{2}}\right)\right) .
$$

Since the hypergeometric function ${ }_{p} F_{p-1}\left(a_{1}, \ldots \ldots a_{p} ; b_{1}, \ldots \ldots . . b_{p-1} ; \sigma z\right)$ can reproduce the same form of large-order behavior of the perturbation series under consideration, it is then recommended as an approximant for the perturbation series of $E_{0}^{J}$ above. Near the tip of the branch cut, the hypergeometric function has a power-law behavior of the form: 


$$
{ }_{p} F_{p-1}\left(a_{1}, \ldots \ldots a_{p} ; b_{1}, \ldots \ldots . . b_{p-1} ; \sigma z\right) \propto(1-\sigma z)^{-\psi},
$$

where $\psi=\sum_{i=1}^{p} a_{i}-\sum_{i=1}^{p-1} b_{i}=b+1$ or in other words, the critical exponent $\psi$ is solely determined by the large-order parameter $b$ which means that this parameter is universal.

Based on the above clarifications, the hypergeometric resummation algorithm can be simplified into two simple steps:

1. Match the available orders from the perturbation series with the corresponding number of terms from the expansion of ${ }_{p} F_{p-1}\left(a_{1}, \ldots \ldots a_{p} ; b_{1}, \ldots \ldots . . b_{p-1} ; \sigma J\right)$.

2. Employ the large order relation

$$
\sum_{i=1}^{p} a_{i}-\sum_{i=1}^{p-1} b_{i}-1=-\frac{3}{2}
$$

in the set of coupled equations to obtain the $b_{i}$ parameters. Note that the $a_{i}$ parameters for the model under consideration are known [26].

Let us give an example for a certain order of the hypergeometric approximant. Assume that we have the second order perturbation series of the form:

$$
Q(z)=c_{0}+c_{1} z+c_{2} z^{2}+O\left(z^{3}\right)
$$

with the large order behavior in Eq.(5) but with $p=q+1$, then the suggested hypergeometric approximant is

$$
Q(z) \sim c_{0}{ }_{3} F_{2}\left(a_{1}, a_{2}, a_{3} ; b_{1}, b_{2} ; \sigma z\right)
$$

$c_{0}{ }_{3} F_{2}\left(a_{1}, a_{2}, a_{3} ; b_{1}, b_{2} ; \sigma z\right)$ has the expansion:

$$
c_{0}{ }_{3} F_{2}\left(a_{1}, a_{2}, a_{3} ; b_{1}, b_{2} ; \sigma z\right)=c_{0}+c_{0} \frac{a_{1} a_{2} a_{3} \sigma}{b_{1} b_{2}} z+c_{0} \frac{a_{1}\left(1+a_{1}\right) a_{2}\left(1+a_{2}\right) a_{3}\left(1+a_{3}\right) \sigma^{2}}{b_{1}\left(1+b_{1}\right) b_{2}\left(1+b_{2}\right)} z^{2}+O\left(z^{3}\right)
$$

Matching this expansion with the series in Eq.(20), we get the following set of equations:

$$
\begin{aligned}
c_{0} \frac{a_{1} a_{2} a_{3} \sigma}{b_{1} b_{2}} & =c_{1}, \\
c_{0} \frac{a_{1}\left(1+a_{1}\right) a_{2}\left(1+a_{2}\right) a_{3}\left(1+a_{3}\right) \sigma^{2}}{b_{1}\left(1+b_{1}\right) b_{2}\left(1+b_{2}\right)} & =c_{2},
\end{aligned}
$$


also the the numerator and the denominator parameters are constrained by the large order relation:

$$
a_{1}+a_{2}+a_{3}-\left(b_{1}+b_{2}\right)-1=b \text {. }
$$

This set of three coupled equations is to be solved for the unknown parameters $b_{1}, b_{2}$ and $\sigma$. Note that the parameter $\sigma$ can be obtained from quasi-classical methods but it is out of the scope of this work.

For the model with the ground state perturbation series in Eq.(13), we have $a_{1}=\frac{-3}{2}$, $a_{2}=\frac{-1}{4}$ and $a_{3}=1$ [26] while the large order parameter $b=-\frac{3}{2}$ [25]. Thus the solution of the above set of equations for that model yields the results $b_{1}=-0.60310956052580091716$, $b_{2}=0.35310956052580091716$ and $\sigma=-0.560266190804551029423$. Accordingly we have the second order approximant:

$$
E_{0}^{J} \simeq 0.37{ }_{3} F_{2}\left(\frac{-3}{2}, \frac{-1}{4}, 1 ;-0.60,0.35 ;-0.56 J\right) .
$$

One can involve more perturbative terms as input by going to ${ }_{4} F_{3},{ }_{5} F_{4} \ldots$ and so on. To test the accuracy of the algorithm, we compare its prediction with exact (numerical results) from Ref.[45, 46] in table I . Note that the vacuum energy for the Hamiltonian in Eq.(10) and that in Eq.(11) are related as $E_{0}^{g}=J^{\frac{-1}{4}}\left(E_{0}^{J}-\frac{1}{3} m^{6} J^{\frac{3}{2}}\right)$ and $J=\frac{i m^{4}}{g^{\frac{4}{5}}}$ while the coupling $\lambda$ in Refs.[45, 46] is related to $g$ by the relation $g=288 \lambda^{2}$. From table I, one can realize that the accuracy of the algorithm is improved form order to order.

One can obtain the edge critical exponent and the critical coupling of the theory by noting that the hypergeometric functions ${ }_{p} F_{p-1}\left(a_{1}, \ldots \ldots a_{p} ; b_{1}, \ldots \ldots . b_{q} ; \sigma z\right)$ have a power-law behavior around the tip of the branch cut (starting from $\sigma z=1$ to $\sigma z \rightarrow \infty$ ) in the form $[37,59,60]$ :

$$
{ }_{p} F_{p-1}\left(a_{1}, . . a_{p} ; b_{1}, . . b_{p-1} ; \sigma z\right)-{ }_{p} F_{p-1}\left(a_{1}, . . a_{p} ; b_{1}, . . b_{p-1} ; 1\right) \propto(1-\sigma z)^{y},
$$

where

$$
y=\sum_{i=1}^{p-1} b_{i}-\sum_{i=1}^{p} a_{i}=-(b+1) .
$$

This means that as $J \rightarrow J_{c}=\frac{1}{\sigma}$ we have

$$
E_{0}^{J}(J)-E_{0}^{J_{c}}=\propto(1-\sigma J)^{\frac{1}{2}} .
$$

The critical coupling $J_{c}$ from the second order approximant in Eq.(22) is thus $J_{c}=-1.7849$ compared to $J_{c}=-1.3510$ from ODM rsummation at the 150th order from Ref.[26]. In fact, 
TABLE I. Comparison of our prediction for $E_{0}^{g}$ and numerical results $E_{\text {exact }}$ from Ref.[45, 46]. We get first the hypergeometric approximations ${ }_{3} F_{2},{ }_{4} F_{3},{ }_{5} F_{4}$ and ${ }_{6} F_{5}$ for the perturbation series of $E_{0}^{J}$ and then transform it to $E_{0}^{g}$. Note that $J=\frac{i m^{4}}{g^{\frac{4}{5}}}$ and $g=288 \lambda^{2}$ while we set $m=1$.

\begin{tabular}{|c|c|c|c|c|l|}
\hline$\lambda$ & ${ }_{3} F_{2}$ & ${ }_{4} F_{3}$ & ${ }_{5} F_{4}$ & ${ }_{6} F_{5}$ & Exact \\
\hline 0.015625 & 0.682387 & 0.504794 & 0.501965 & 0.502697 & 0.502621 \\
\hline 0.03125 & 0.534941 & 0.510201 & 0.509934 & 0.509978 & 0.50998 \\
\hline 0.0625 & 0.536264 & 0.533944 & 0.533931 & 0.533932 & 0.53393 \\
\hline 0.125 & 0.595069 & 0.594916 & 0.594915 & 0.594915 & 0.59492 \\
\hline 0.25 & 0.712944 & 0.712936 & 0.712936 & 0.712936 & 0.71294 \\
\hline 0.5 & 0.900258 & 0.900258 & 0.900258 & 0.900258 & 0.90026 \\
\hline 1 & 1.16745 & 1.16745 & 1.16745 & 1.16745 & 1.16746 \\
\hline 2 & 1.53077 & 1.53077 & 1.53077 & 1.53077 & 1.53078 \\
\hline
\end{tabular}

at the fifth order approximant $\left({ }_{6} F_{5}\right)$ we obtained a precise value for the critical coupling $J_{c}$ as shown in table II.

TABLE II. The hypergeometric ${ }_{3} F_{2},{ }_{4} F_{3},{ }_{5} F_{4}$ and ${ }_{6} F_{5}$ predictions for the critical coupling $J_{c}$ compared to the $150^{\text {th }}$ order of the ODM method in Ref.[26]. All approximants predict the same exact critical exponents as shown because they depend solely on the large order parameter $b=-3 / 2$. The fifth order approximant $\left({ }_{6} F_{5}\right)$ gives a very precise critical coupling as shown in the table.

\begin{tabular}{|c|c|c|c|c|}
\hline Approximant & $J_{c}$ & $\nu_{c}$ & $\delta$ & $\gamma$ \\
\hline${ }_{3} F_{2}$ & -1.78487 & $1 / 2$ & -2 & $3 / 2$ \\
\hline${ }_{4} F_{3}$ & -1.30267 & $1 / 2$ & -2 & $3 / 2$ \\
\hline${ }_{5} F_{4}$ & -1.32908 & $1 / 2$ & -2 & $3 / 2$ \\
\hline${ }_{6} F_{5}$ & -1.35062 & $1 / 2$ & -2 & $3 / 2$ \\
\hline ODM & -1.3510 & - & - & - \\
\hline
\end{tabular}

What is really impressive is that according to our prediction, the critical exponent $\nu_{c}=\frac{1}{2}$ which is extracted from the relation $\left(E_{0}^{J}(J)-E_{0}^{J_{c}}\right) \propto(1-\sigma J)^{d \nu_{c}}[61]$. This result is exact [42] $(d=1)$ and does not depend on the order of approximation but on the other hand depends solely on the large order parameter $b=-\frac{3}{2}$. This is clear from Eq.(19) where we 
find $y=-1-\left(-\frac{3}{2}\right)=\frac{1}{2}$. It has been shown in Ref.[42] that the edge critical exponent $\nu_{c}$ for one dimensional Ising model is $\nu_{c}=\frac{1}{2}$ exactly the same value we obtained. Note that here we used the scaling relation $\left(E_{0}^{J}(J)-E_{0}^{J_{c}}\right) \propto \zeta_{\text {gap }}^{-d} \propto\left(J-J_{c}\right)^{-d \nu_{c}}[61]$, where $\zeta_{\text {gap }}^{-d}$ is the correlation length. Up to the best of our knowledge this is the first time to get exact critical exponents from only the knowledge of the large-order parameters. Note that the square root singularity of the ground state energy near the critical coupling $J_{c}$ has been suggested based on the analysis of the calculations in Ref.[26] but here we get it exactly.

The ground state energy or equivalently the effective potential is well known to be the generating functional of the one-particle irreducible amplitudes [62]. Accordingly, one can obtain other amplitudes like magnetization (vacuum expectation value) and magnetic susceptibility for instance from successive differentiation with respect to $\left(\frac{1}{2} i J\right)$. Thus the vacuum condensate $v$ is given by

$$
v=\frac{\partial E_{0}^{J}}{\partial\left(\frac{1}{2} i J\right)}
$$

where the hypergeometric approximant for $E_{0}^{J}$ is given by

$$
E_{0}^{J} \approx c_{0}{ }_{p} F_{p-1}\left(a_{1}, \ldots \ldots a_{p} ; b_{1}, \ldots \ldots . . b_{q} ; \sigma J\right)
$$

Note also that

$$
\frac{\partial}{\partial z}{ }_{p} F_{q}\left(a_{1}, \ldots \ldots . . a_{p} ; b_{1} \ldots \ldots, b_{q} ; z\right)=\frac{\prod_{j=1}^{p} a_{j}}{\prod_{j=1}^{q} b_{j}} F_{q}\left(a_{1}+1, \ldots \ldots a_{p}+1 ; b_{1}+1, \ldots . b_{q}+1 ; z\right) .
$$

We found that the vacuum expectation value is negative imaginary as it is well known for such $\mathcal{P} \mathcal{T}$-symmetric model [63]. The derivative of the hypergeometric function is thus another hypergeometric function but with every numerator and denominator parameter is increased by 1 . The exponent of the power-law behavior of the derivative will thus decrease by 1 . Thus near the critical point, the vacuum condensate $v$ has a power-law behavior of the from:

$$
v(J)-v\left(J_{c}\right) \propto\left(J-J_{c}\right)^{\frac{1}{\delta}}
$$

where $\frac{1}{\delta}=y-1$ and $y$ is defined in Eq.(24). Since we obtained $y=\frac{1}{2}$ for the model under consideration, we get $\delta=-2$. This is again the exact exponent reported in Ref. [42].

The magnetic susceptibility $\chi$ is given by:

$$
\chi=\frac{\partial^{2} E_{0}^{J}}{\partial\left(\frac{1}{2} i J\right)^{2}} .
$$


Accordingly, $\chi$ has the power-law behavior

$$
\chi \propto\left(J-J_{c}\right)^{-\gamma}, \quad-\gamma=y-2=\frac{-3}{2}
$$

This result is in accordance with scaling relations that relate $\gamma$ to $\delta$ as [64]

$$
-\gamma=\frac{1-\delta}{\delta}=\frac{-3}{2}
$$

So again our result is exact. Since all critical exponents here are obtained from the largeorder parameter $b$, the results suggest the universality of such parameter.

\section{HYPERGEOMETRIC APPROXIMATION FOR THE HT-EXPANSION OF THE SUSCEPTIBILITY WITHIN THE SC LATTICE}

In the previous section, we considered a $0+1$ dimensional quantum field example for which the parameter $b$ is known exactly and showed that the critical exponent is solely determined by $b$. To test the validity of our conjecture for a higher dimensional example, one should first note that the mathematical structure of the strong-coupling expansion of the $\phi^{4}$ lattice field theory is equivalent to the HT-expansion of the Ising model [11]. With no loss of generality, for a field theory in higher dimensions, one can consider the high-temperature expansion within the Ising model to test the universality of the large-order parameter $b$. Up to the best of our knowledge, the large-order asymptotic behavior is not known exactly for such expansion. However, we choose such a case because the expansion is known up to high order and thus an accurate prediction of $b$ can be obtained using the hypergeometric approximants. Since the high temperature expansion within the Ising model has a finite radius of convergence, the expanded quantity can be approximated by the hypergeometric approximant ${ }_{p} F_{p-1}\left(a_{1}, \ldots \ldots a_{p} ; b_{1}, \ldots \ldots . . b_{q} ; \sigma z\right)$ which has the large-order asymptotic behavior of the form $\sigma^{n} n^{b}\left(1+O\left(\frac{1}{n}\right)\right)$. A very good approximation of the parameters $b$ and $\sigma$ can be extracted from the available many terms of the perturbation series. The HT-expansion up to $O\left(\beta^{25}\right)$ for the susceptibility of the $S C$ lattice $\left(S=\frac{1}{2}\right)$ is given by [6]: 


$$
\begin{aligned}
\chi(\beta) & =\sum_{i=0}^{\infty} c_{i} \beta^{i}=1+6 \beta+30 \beta^{2}+148 \beta^{3}+706 \beta^{4}+\ldots \ldots \ldots \\
& +\frac{93592219478518291774477772}{3093594879375} \beta^{20} \\
& +\frac{8972803527064109944099241768}{/ 64965492466875} \beta^{21} \\
& +\frac{5296430224856866468505272024}{8407299025125} \beta^{22} \\
& +\frac{47221618622049399307213422740992}{16436269594119375} \beta^{23} \\
& +\frac{37975296352037116774213386661036}{2900518163668125} \beta^{24} \\
& +\frac{73538934029908819825899053186296808}{1232720219558953125} \beta^{25}+\ldots \ldots
\end{aligned}
$$

One can go and parametrizes the approximant ${ }_{p} F_{p-1}\left(a_{1}, \ldots \ldots a_{p} ; b_{1}, \ldots \ldots . . b_{q} ; \sigma z\right)$ starting from the lowest orders and watch the convergence as more orders are employed. However, according to our conjecture the critical indices are encoded in the large orders. Accordingly, to obtain fast accurate predictions for the large-order parameters $b$ and $\sigma$, it would be better to parametrize the the approximant ${ }_{p} F_{p-1}\left(a_{1}, \ldots \ldots a_{p} ; b_{1}, \ldots \ldots . . b_{q} ; \sigma z\right)$ starting from highest to lower orders. Also, as explained in Ref.[7], the oscillation in the sign of the term $O\left(\frac{1}{n}\right)$ in the large-order form demands us to treat odd (or even) terms separately. Accordingly, the parametrization of the approximant can go through matching the relations (for odd terms)

$$
\begin{aligned}
R_{n}^{o d d} & =\frac{c_{i}}{c_{i-2}}, i=25,23,21, \ldots \ldots \\
R_{25} & =\frac{c_{25}}{c_{23}}, R_{23}=\frac{c_{23}}{c_{21}}, R_{21}=\frac{c_{21}}{c_{19}}, \ldots
\end{aligned}
$$

For instance, the four parameters approximant $\chi \simeq{ }_{2} F_{1}\left(a_{1}, a_{2} ; b_{1} ; \sigma z\right)$ can be parametrized as:

$$
\begin{aligned}
& \frac{\left(a_{1}+23\right)\left(a_{1}+24\right)\left(a_{2}+23\right)\left(a_{2}+24\right)}{\left(b_{1}+23\right)\left(b_{1}+24\right)} \sigma^{2}=\frac{9192366753738602478237381648287101}{737837790969521864175209730328} \\
& \frac{\left(a_{1}+21\right)\left(a_{1}+22\right)\left(a_{2}+21\right)\left(a_{2}+22\right)}{\left(b_{1}+21\right)\left(b_{1}+22\right)} \sigma^{2}=\frac{11805404655512349826803355685248}{1121600440883013743012405221} \\
& \frac{\left(a_{1}+19\right)\left(a_{1}+20\right)\left(a_{2}+19\right)\left(a_{2}+20\right)}{\left(b_{1}+19\right)\left(b_{1}+20\right)} \sigma^{2}=\frac{4486401763532054972049620884}{512422018420605679707007} \\
& \frac{\left(a_{1}+17\right)\left(a_{1}+18\right)\left(a_{2}+17\right)\left(a_{2}+18\right)}{\left(b_{1}+17\right)\left(b_{1}+18\right)} \sigma^{2}=\frac{2049688073682422718828028}{286754736324335778241}
\end{aligned}
$$


Solving these equations for the parameters $a_{1}, a_{2}, b_{1}$ and $\sigma$ gives the result:

$$
\begin{gathered}
\sigma=4.5108, \quad a_{1}=-23.2765-1.20827 \times 10^{-8} i \\
a_{2}=1.24944, \quad b_{1}=-23.2765-1.20826 \times 10^{-8} i .
\end{gathered}
$$

The large-order parameter $b$ is related to the numerator parameters $a_{i}$, and the denominator parameter $b_{i}$ as:

$$
\begin{aligned}
b & =\sum_{i=1}^{p} a_{i}-\sum_{i=1}^{p-1} b_{i}-1 \\
& =0.2494-1.0 \times 10^{-13} i \\
& \simeq 0.2494 .
\end{aligned}
$$

Thus the $\gamma$ exponent is given by $\gamma=b+1=1.2494$ compared to 1.237 in Ref.[7]. Also, the critical inverse temperature $\beta_{c}=\frac{1}{\sigma}=0.22161$ compared to 0.22165 from Ref.[7]. So again the large order parameter $b$ determines the critical exponent. Note that, very close results are obtained by treating the even terms but we prefer the odd ones as the last term is odd and it is assumed to be more effective in determining the large-order parameter $b$. The six parameters approximant $\chi \simeq{ }_{3} F_{2}\left(a_{1}, a_{2}, a_{3} ; b_{1}, b_{2} ; \sigma z\right)$ can also be parametrized through the following relations:

$$
\begin{aligned}
& \frac{\left(a_{1}+23\right)\left(a_{1}+24\right)\left(a_{2}+23\right)\left(a_{2}+24\right)\left(a_{3}+23\right)\left(a_{3}+24\right)}{\left(b_{1}+23\right)\left(b_{1}+24\right)\left(b_{2}+23\right)\left(b_{2}+24\right)} \sigma^{2}=20.7642 \\
& \frac{\left(a_{1}+21\right)\left(a_{1}+22\right)\left(a_{2}+21\right)\left(a_{2}+22\right)\left(a_{3}+21\right)\left(a_{3}+22\right)}{\left(b_{1}+21\right)\left(b_{1}+22\right)\left(b_{2}+21\right)\left(b_{2}+22\right)} \sigma^{2}=20.801382 \\
& \frac{\left(a_{1}+19\right)\left(a_{1}+20\right)\left(a_{2}+19\right)\left(a_{2}+20\right)\left(a_{3}+19\right)\left(a_{3}+20\right)}{\left(b_{1}+19\right)\left(b_{1}+20\right)\left(b_{2}+19\right)\left(b_{2}+20\right)} \sigma^{2}=20.845921 \\
& \frac{\left(a_{1}+17\right)\left(a_{1}+18\right)\left(a_{2}+17\right)\left(a_{2}+18\right)\left(a_{3}+17\right)\left(a_{3}+18\right)}{\left(b_{1}+17\right)\left(b_{1}+18\right)\left(b_{2}+17\right)\left(b_{2}+18\right)} \sigma^{2}=20.900230 \\
& \frac{\left(a_{1}+15\right)\left(a_{1}+16\right)\left(a_{2}+15\right)\left(a_{2}+16\right)\left(a_{3}+15\right)\left(a_{3}+16\right)}{\left(b_{1}+15\right)\left(b_{1}+16\right)\left(b_{2}+15\right)\left(b_{2}+16\right)} \sigma^{2}=20.967904 \\
& \frac{\left(a_{1}+13\right)\left(a_{1}+14\right)\left(a_{2}+13\right)\left(a_{2}+14\right)\left(a_{3}+13\right)\left(a_{3}+14\right)}{\left(b_{1}+13\right)\left(b_{1}+14\right)\left(b_{2}+13\right)\left(b_{2}+14\right)} \sigma^{2}=21.054549 .
\end{aligned}
$$


The solution of this set of equations gives the values:

$$
\begin{aligned}
& \sigma=4.51093, \quad a_{1}=-15.4176-2.76717 \times 10^{-9} i \\
& a_{2}=1.24892, \quad a_{3}=-17.7923-2.96486 \times 10^{-9} i \\
& b_{1}=-15.4176-2.76719 \times 10^{-9} i \\
& b_{2}=-17.7923-2.9648 \times 10^{-9} i .
\end{aligned}
$$

This gives $b=0.2489-3.76237 \times 10^{-14} i$ and $\beta_{c}=0.22168$. So again the large order parameter $b$ determines the critical exponent. Also, for the eight parameters hypergeometric approximants ${ }_{4} F_{3}\left(a_{1}, a_{2}, a_{3}, a_{4} ; b_{1}, b_{2}, b_{3} ; \sigma \beta\right)$, we obtained the following parameters values:

$$
\begin{aligned}
& \sigma=4.51101, \quad a_{1}=-15.8686-5.10971 \times 10^{--7} i \\
& a_{2}=1.2483819+2.37927 \times 10^{-10} i, a_{3}=-11.3347-4.45387 \times 10^{-8} i \\
& a_{4}=-13.6312-2.11442 \times 10^{-7} i, b_{1}=-11.3345-4.46736 \times 10^{-8} i, \\
& b_{2}=-13.6314-2.11099 \times 10^{-7} i, b_{3}=-15.8686-5.11116 \times 10^{-7} i,
\end{aligned}
$$

This leads to the result $b=0.24838+1.74976 \times 10^{\wedge}-10 i$ and $\beta_{c}=\frac{1}{4.51101}=0.22168$. In table III, we listed the hypergeometric predictions for $b, \gamma$ and $\beta_{c}$.

TABLE III. The hypergeometric approximants ${ }_{2} F_{1},{ }_{3} F_{2}$ and ${ }_{4} F_{3}$ predictions for the large-order parameters $b$ and $\beta_{c}=\frac{1}{\sigma}$ of the HT-series expansion of the susceptibility of the SC lattice. These approximants are parametrized using the last odd orders of the series. Also, the critical exponent $\gamma=b+1$ is listed where it can be compared to $\gamma=1.237$ in Ref.[7] while the critical inverse temperature $\beta_{c}$ can be compared with $\beta_{c}=0.22165$ from the same reference.

\begin{tabular}{|c|c|c|c|}
\hline Approximant & $b$ & $\gamma$ & $\beta_{c}$ \\
\hline${ }_{2} F_{1}$ & 0.2494 & 1.2494 & 0.22169 \\
\hline${ }_{3} F_{2}$ & 0.2489 & 1.2489 & 0.22168 \\
\hline${ }_{4} F_{3}$ & 0.24838 & 1.24838 & 0.22168 \\
\hline
\end{tabular}

Note that the series in Eq.(25) is not alternating in sign and thus the hypergeometric approximant is suffering from a problem like non-Borel summability. In fact the hypergeometric approximant has a branch cut starts from $\beta=1 / \sigma$ to infinity. This means that it can (accurately) describe the high temperature phase only. For the low temperature phase, 
the hypergeometric approximants suffer from the existence of Stokes phenomena which can be cured [65] but it is out of the scope of this work.

\section{HYPERGEOMETRIC APPROXIMATION FOR THE HT-EXPANSION OF THE SUSCEPTIBILITY OF THE SQ ISING MODEL}

The HT-expansion for the susceptibility of the spin-half square lattice is given by [6]:

$$
\begin{aligned}
\chi(\beta) & =1+4 \beta+12 \beta^{2}+\frac{104}{3} \beta^{3}+92 \beta^{4}+\ldots \ldots \ldots \\
& +\frac{415782048556042942544}{3093594879375} \beta^{20}+\frac{4735391065845611373232}{14992036723125} \beta^{21} \\
& +\frac{529562920319138348552816}{714620417135625} \beta^{22}+\frac{85616154520095267692857616}{49308808782358125} \beta^{23} \\
& +\frac{66773068948180944546678128}{16436269594119375} \beta^{24}+\frac{3192145249472459217984684656}{336196423516078125} \beta^{25}+\ldots \ldots,
\end{aligned}
$$

As long as we are interested only in large-order parameters, one can fit the ratio $R_{n}=\frac{C_{n}}{C_{n-1}}$ of the above series with the corresponding ratio of expansion coefficients of the hypergeometric approximant but for large orders. In fact, if this series to have a finite radius of convergence, then for large $n$ it has to fit the corresponding ratio from the expansion of the hypergeometric approximant as:

$$
\begin{aligned}
R_{n} & \simeq \frac{(\sigma)^{n} n^{b}}{(\sigma)^{n-1}(n-1)^{b}} \\
& =\sigma \frac{1}{\left(1-\frac{1}{n}\right)^{b}} \\
& \simeq \sigma\left(1+b\left(\frac{1}{n}\right)\right)
\end{aligned}
$$

In Fig.4, we plot the ratio $R_{n}$ versus $\frac{1}{n}$ from which we obtained the large order parameters values $b=0.75033$ and $\sigma=2.269$. These values lead to the critical exponent $\gamma=b+$ $1=1.75033$ and critical $\beta$ as $\beta_{c}=\frac{1}{\sigma}=0.44072$. The exact values are well known to be $\gamma=\nu(2-\eta)=1.75$ [66],$\beta_{c}=0.4407$ [67]. Again, this example assures the universality of the large-order parameter $b$ for the HT series expansion. 


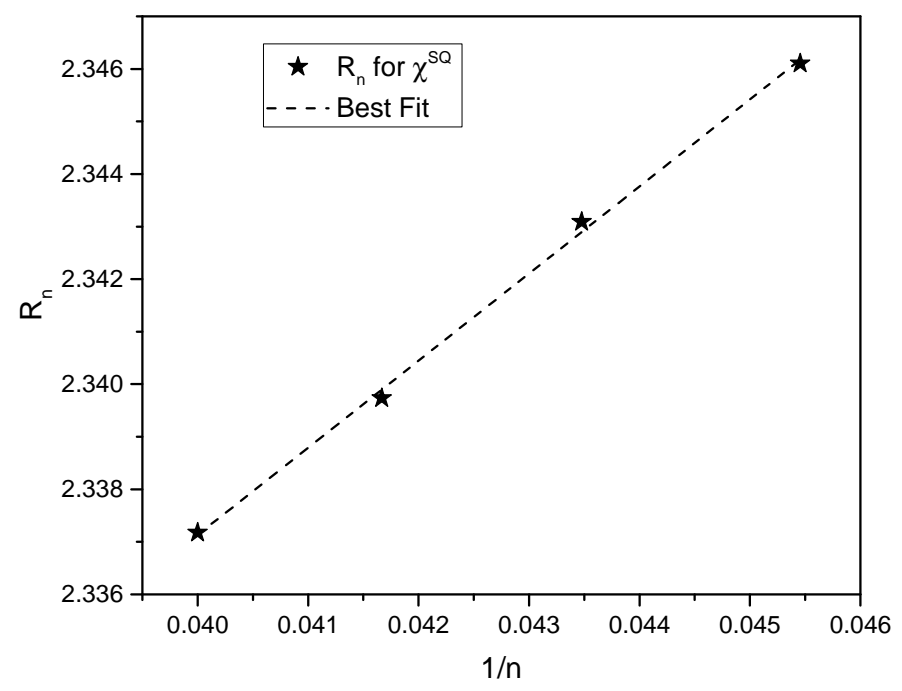

FIG. 4. In this figure, we plot the ratio $R_{n}$ for the HT series expansion of the susceptibility of the spin-half square lattice at large orders. The data has a straight line fit of the form $R_{n}=$ $1.7025(1 / n)+2.269$ which predicts the values $\sigma=2.269$ and $b=0.75033$.

\section{SUMMARY AND CONCLUSIONS}

Near the tip of the branch cut, the hypergeometric functions ${ }_{k+1} F_{k}$ have a power-law behavior similar to a physical quantity (magnetization for instance) near second-order phase transition where it follows the form $Q(\beta) \propto\left(1-\frac{\beta}{\beta_{c}}\right)^{\psi}$. The exponent $\psi$ has been proven to follow the relation $\psi=-(b+1)$, where $b$ is the large-order parameter of the series. This link between $b$ and $\psi$ is in complete agreement with the theorem of Darboux. Accordingly, one can determine the exact critical exponent provided that we know the large-order parameter $b$ of a series expansion with finite radius of convergence. For the weak coupling expansion which is generated around an essential singularity, Darboux theorem is not applicable and thus a direct link between $\psi$ and $b$ is missing. On the other hand, the strong-coupling (HT) expansion is well known to have a finite radius of convergence. Accordingly, one can expect that the large-order parameter $b$ of the strong-coupling (HT) expansion is universal. In fact there are techniques in quantum field theory that might enable us to get exact values for the parameter $b$ and in this case exact critical exponents can be extracted.

We tested our conjecture about universality of $b$ using the Yang-Lee model in $0+1$ space- 
time dimensions as well as the HT-series expansion for Ising model. The strong coupling expansion of the Bose-Hubbard model is agreeing with our thoughts too [60, 68].

We started from the large-order behavior of the perturbation series of the ground state energy of the Yang-Lee model to select the suitable hypergeometric approximant. We realized that the large-order behavior does not have a growth factor $n$ ! and found that the hypergeometric functions ${ }_{p} F_{p-1}$ do have the same form of the given large-order behavior. This recommends them to be the most suitable hypergeometric approximants for the given series. We set a constraint on the numerator and denominator parameters based on matching both large-order behaviors. The large-order constraint on the parameters has accelerated the convergence which has been tested by calculating up to fifth order approximants $\left({ }_{6} F_{5}\right)$ and found that they yield very precise predictions of the ground state energy compared to exact (numerical) results from literature.

$\mathcal{P} \mathcal{T}$ - symmetry breaking has been investigated by noting that the hypergeometric functions ${ }_{p} F_{p-1}\left(a_{1}, a_{2}, \ldots . a_{p} ; b_{1}, b-2, \ldots b_{p-1} ;-\sigma z\right)$ have branch cut starting at $-\sigma z=1$ and extends to $-\sigma z=\infty$. Near the branch point $-\sigma z=1$, the hypergeometric function has a power-law behavior from which we were able to get the exact $\nu_{c}$ critical exponent and a very precise value for the critical coupling. We found that the exact critical exponent can be extracted from the large-order parameter $b$ which up to the best of our knowledge is the first time to extract exact critical exponents directly from large-order parameters. This prediction might open the door to directly extract exact critical exponents from obtaining the large-order behavior of a given perturbation series (strong-coupling (HT)).

Since the ground state energy serves as the effective potential, it enables us to obtain all the critical exponents (exact) by successive differentiation of the ground state energy (effective potential) with respect to the external magnetic field.

The critical exponents of the Yang-Lee model are always stressed within the investigation of the zeros of the partition function which very recently has been exposed to experimental investigations [40]. In this work we extracted the edge exponent $\delta$ from the dependance of the order parameter on the external magnetic field. In Ref.[43], we have shown that the critical point of $\mathcal{P} \mathcal{T}$ - symmetry breaking is in fact a Yang-Lee edge singularity. So our results here might lead to experimental investigation of Yang-Lee edge singularity via testing $\mathcal{P} \mathcal{T}$-symmetry breaking as well as watching the behavior of the order parameter near the point of symmetry breaking. 
Our conjecture has been tested also by considering the HT-series expansion of the SC and SQ lattices of the spin half Ising model. From the many orders known for susceptibility expansion, we were able to get accurate values for the large-order parameter $b$. The universality of $b$ has been also assured for these examples by comparing the extracted critical exponent with the well known one of the Ising model. We also obtained a very accurate critical temperature from the approximate large-order behavior of the given series.

The main message of this work is to draw the attention to the importance of determining the large-order asymptotic behavior of the strong-coupling (HT) expansion in field theory. There exists well known methods to do that for a series expansion and thus if we were able to get exact values for $b$, it will lead to the first determination of an exact critical exponent in three dimensions. For the $O(2)$ symmetric $\phi^{4}$ theory this will resolve the current $\lambda$-point dispute between theory and experiment [69-71].

[1] F. J. Dyson, Divergence of Perturbation Theory in Quantum Electrodynamics, Phys. Rev. 85, $631(1952)$.

[2] J. P. Boyd, The Devil's Invention: Asymptotic, Superasymptotic and Hyperasymptotic Series, Acta Appl. Math. 56, 10.1023/A:1006145903624 (1999).

[3] H. Kleinert, Path Integrals in Quantum Mechanics, Statistics, Polymer Physics, and Financial Markets (WORLD SCIENTIFIC, 2009).

[4] C. M. Bender, F. Cooper, G. S. Guralnik, and D. H. Sharp, Strong-coupling expansion in quantum field theory, Phys. Rev. D 19, 1865 (1979).

[5] W. Janke and H. Kleinert, Convergent Strong-Coupling Expansions from Divergent WeakCoupling Perturbation Theory, Phys. Rev. Lett. 75, 2787 (1995).

[6] P. Butera and M. Comi, An On-Line Library of Extended High-Temperature Expansions of Basic Observables for the Spin-S Ising Models on Two- and Three-Dimensional Lattices, J. Stat. Phys. Vol. 109, https://doi.org/10.1023/A:1019995830014 (2002).

[7] P. Butera and M. Comi, Critical universality and hyperscaling revisited for Ising models of general spin using extended high-temperature series, Phys. Rev. B 65, 144431 (2002).

[8] G. Jug and B. N. Shalaev, Duality symmetry, strong-coupling expansion and universal critical amplitudes in two-dimensional Phi 4 field models, J. Phys. A. Math. Gen. 32, 7249 (1999). 
[9] G. A. Baker, L. Benofy, F. Cooper, and D. Preston, Analysis of the lattice, strong coupling series for $g_{0} \phi^{4}$ field theory in d dimensions, Nucl. Phys. B 210, 273 (1982).

[10] F. Cooper, B. Freedman, and D. Preston, Solving $\phi_{1,2}^{4}$ field theory with Monte Carlo, Nucl. Phys. B 210, 210 (1982).

[11] H. Yamada, Delta expansion on the lattice and dilated scaling region, Phys. Rev. D 76, 045007 (2007).

[12] J. Langelage, G. Münster, and O. Philipsen, Strong coupling expansion for Yang-Mills theory at finite temperature, PoS LAT2007201,2007 (2007), arXiv:0710.0512.

[13] A. M. Shalaby, Precise critical exponents of the $O(N)$-symmetric quantum field model using hypergeometric-Meijer resummation, Phys. Rev. D 101, 105006 (2020).

[14] L. Berg, R. B. Dingle, Asymptotic Expansions: Their Derivation and Interpretation. XV +521 S. m. Fig. London/New York 1973. Academic Press. Preis geb. £ 13.50, ZAMM - Zeitschrift für Angew. Math. und Mech. 54, 520 (1974).

[15] P. Butera and M. Comi, 2n-point renormalized coupling constants in the three-dimensional Ising model:Estimates by high temperature series to order $\beta^{17}$, Phys. Rev. E 55, 6391 (1997).

[16] C. M. Bender and S. Boettcher, Dimensional expansion for the Ising limit of quantum field theory, Phys. Rev. D 48, 4919 (1993).

[17] M. Campostrini, A. Pelissetto, P. Rossi, and E. Vicari, Two-point correlation function of three-dimensional $O(N)$ models: The critical limit and anisotropy, Phys. Rev. E 57, 184 (1998).

[18] H. Arisue and T. Fujiwara, High order calculation of the strong coupling expansion for the mass gap in lattice gauge theory, Nucl. Phys. B 285, 253 (1987).

[19] N. Svaiter, The strong-coupling expansion and the ultra-local approximation in field theory, Phys. A Stat. Mech. its Appl. 345, 517 (2005).

[20] A. M. Shalaby, Weak-Coupling, Strong-Coupling and Large-Order Parametrization of the Hypergeometric-Meijer Approximants, Results Phys. 19, 103376 (2020), arXiv:2002.05110.

[21] A. M. Shalaby, Critical exponents of the $O(N)$-symmetric $\phi^{4}$ model from the $\varepsilon^{7}$ hypergeometric-Meijer resummation, Eur. Phys. J. C 81, 87 (2021), arXiv:2005.12714.

[22] H. Kleinert and V. Schulte-Frohlinde, Critical Properties of $\phi^{4}$-Theories (WORLD SCIENTIFIC, 2001).

[23] Y. Nishiyama, Strong-coupling-expansion analysis of the false-vacuum decay rate of the lattice 
$\phi^{4}$ model in $1+1$ dimensions, J. Phys. A. Math. Gen. 34, 11215 (2001).

[24] M. Campostrini, M. Hasenbusch, A. Pelissetto, P. Rossi, and E. Vicari, Critical behavior of the three-dimensional XY universality class, Phys. Rev. B 63, 214503 (2001).

[25] L. Skála, J. Cízek, and J. Zamastil, Strong coupling perturbation expansions for anharmonic oscillators. Numerical results, J. Phys. A. Math. Gen. 32, 5715 (1999).

[26] J. Zinn-Justin and U. D. Jentschura, Imaginary cubic perturbation: numerical and analytic study, J. Phys. A Math. Theor. 43, 425301 (2010).

[27] J. Zinn-Justin, Quantum Field Theory and Critical Phenomena (Oxford University Press, 2002).

[28] M. V. Kompaniets and E. Panzer, Minimally subtracted six-loop renormalization of $O(n)-$ symmetric $\phi^{4}$ theory and critical exponents, Phys. Rev. D 96, 36016 (2017).

[29] R. Guida and J. Zinn-Justin, Critical exponents of the N-vector model, J. Phys. A. Math. Gen. 31, 8103 (1998).

[30] J. A. Gracey, Four loop renormalization of $\phi^{3}$ theory in six dimensions, Phys. Rev. D 92, $025012(2015)$.

[31] J. Zinn-Justin, Precise determination of critical exponents and equation of state by field theory methods, Phys. Rep. 344, 159 (2001).

[32] S. A. Antonenko and A. I. Sokolov, Critical exponents for a three-dimensional $O(n)$-symmetric model with $n>3$, Phys. Rev. E 51, 1894 (1995).

[33] J. C. Le Guillou and J. Zinn-Justin, Critical Exponents for the $n$-Vector Model in Three Dimensions from Field Theory, Phys. Rev. Lett. 39, 95 (1977).

[34] H. Mera, T. G. Pedersen, and B. K. Nikolić, Nonperturbative Quantum Physics from LowOrder Perturbation Theory, Phys. Rev. Lett. 115, 143001 (2015).

[35] A. M. Shalaby, Extrapolating the precision of the Hypergeometric Resummation to Strong couplings with application to the $\mathcal{P} \mathcal{T}$-Symmetric $i \phi^{3}$ Field Theory, Int. J. Mod. Phys. A 35, 2050041 (2018), arXiv:1811.10998.

[36] H. Mera, T. G. Pedersen, and B. K. Nikolić, Fast summation of divergent series and resurgent transseries from Meijer-G approximants, Phys. Rev. D 97, 105027 (2018).

[37] H. Bateman, HIGHER TRANSCENDENTAL FUNCTIONS, Volume I (McGRAW-HILL BOOK COMPANY, INC., 1953).

[38] C. N. Yang and T. D. Lee, Statistical Theory of Equations of State and Phase Transitions. I. 
Theory of Condensation, Phys. Rev. 87, 404 (1952).

[39] T. D. Lee and C. N. Yang, Statistical Theory of Equations of State and Phase Transitions. II. Lattice Gas and Ising Model, Phys. Rev. 87, 410 (1952).

[40] A. Deger and C. Flindt, Determination of universal critical exponents using Lee-Yang theory, Phys. Rev. Res. 1, 023004 (2019).

[41] A. M. Shalaby, Novel phase in the phase structure of the $\left(g \phi^{4}+h \phi^{6}\right)_{1+1}$ field theoretic model, Phys. Rev. D 76, 041702 (2007).

[42] M. E. Fisher, Yang-Lee Edge Singularity and $\phi^{3}$ Field Theory, Phys. Rev. Lett. 40, 1610 (1978).

[43] A. M. Shalaby, Effective action study of the $\mathcal{P} \mathcal{T}$-symmetric $\left(i \phi^{3}\right)_{6-\epsilon}$ theory and the Yang-Lee edge singularity, Int. J. Mod. Phys. A 34, 1950090 (2019).

[44] A. M. Shalaby, Vacuum structure and PT-symmetry breaking of the non-Hermetian $\left(i \phi^{3}\right)$ theory, Phys. Rev. D 96, 025015 (2017).

[45] C. M. Bender and S. Boettcher, Real Spectra in Non-Hermitian Hamiltonians Having $\mathcal{P} \mathcal{T}$ Symmetry, Phys. Rev. Lett. 80, 5243 (1998).

[46] C. M. Bender, S. Boettcher, and P. N. Meisinger, PT-symmetric quantum mechanics, J. Math. Phys. 40, 2201 (1999).

[47] C. M. Bender, V. Branchina, and E. Messina, Ordinary versus $\mathcal{P} \mathcal{T}$-symmetric $\phi^{3}$ quantum field theory, Phys. Rev. D 85, 085001 (2012).

[48] C. M. Bender, V. Branchina, and E. Messina, Critical behavior of the $\mathcal{P} \mathcal{T}$-symmetric $i \phi^{3}$ quantum field theory, Phys. Rev. D 87, 085029 (2013).

[49] Y. Ashida, S. Furukawa, and M. Ueda, Parity-time-symmetric quantum critical phenomena, Nat. Commun. 8, 15791 (2017).

[50] X. Tan, Y. Zhao, Q. Liu, G. Xue, H. Yu, Z. D. Wang, and Y. Yu, Realizing and manipulating space-time inversion symmetric topological semimetal bands with superconducting quantum circuits, npj Quantum Mater. 2, 60 (2017).

[51] S. Pendharker, Y. Guo, F. Khosravi, and Z. Jacob, PT-symmetric spectral singularity and negative-frequency resonance, Phys. Rev. A 95, 033817 (2017).

[52] L. Ge and L. Feng, Contrasting eigenvalue and singular-value spectra for lasing and antilasing in a $\mathcal{P} \mathcal{T}$-symmetric periodic structure, Phys. Rev. A 95, 013813 (2017).

[53] Z. Zhang, Y. Zhang, J. Sheng, L. Yang, M.-A. Miri, D. N. Christodoulides, B. He, Y. Zhang, 
and M. Xiao, Observation of Parity-Time Symmetry in Optically Induced Atomic Lattices, Phys. Rev. Lett. 117, 123601 (2016).

[54] G. Mussardo, Statistical Field Theory: An Introduction to Exactly Solved Models in Statistical Physics (Oxford University Press, 2020).

[55] J. L. Cardy, Conformal Invariance and the Yang-Lee Edge Singularity in Two Dimensions, Phys. Rev. Lett. 54, 1354 (1985).

[56] J. L. Cardy and G. Mussardo, S-matrix of the Yang-Lee edge singularity in two dimensions, Phys. Lett. B 225, 275 (1989).

[57] A. Wipf, Statistical Approach to Quantum Field Theory, Lecture Notes in Physics, Vol. 100 (Springer Berlin Heidelberg, Berlin, Heidelberg, 2013).

[58] A. Laforgia and P. Natalini, On the asymptotic expansion of a ratio of gamma functions, J. Math. Anal. Appl. 389, 833 (2012).

[59] functions.wolfram.com.

[60] S. Sanders, C. Heinisch, and M. Holthaus, Hypergeometric analytic continuation of the strongcoupling perturbation series for the 2d Bose-Hubbard model, EPL (Europhysics Lett. 111, $20002(2015)$.

[61] M. Kaku, Quantum Field Theory A Modern Introduction (Oxford University Press, 1994).

[62] M. Peskin and D. Schroeder, An Introduction to quantum field theory (Addison-Wesley, Reading, USA, 1995).

[63] C. M. Bender, P. N. Meisinger, and H. Yang, Calculation of the one-point Green's function for a $-g \phi^{4}$ quantum field theory, Phys. Rev. D 63, 045001 (2001).

[64] A. Pelissetto and E. Vicari, Critical phenomena and renormalization-group theory, Phys. Rep. 368, 549 (2002).

[65] R. Paris, Smoothing of the Stokes phenomenon using Mellin-Barnes integrals, J. Comput. Appl. Math. 41, 117 (1992).

[66] L. Onsager, Crystal Statistics. I. A Two-Dimensional Model with an Order-Disorder Transition, Phys. Rev. 65, 117 (1944).

[67] F. J. Wegner, Duality in generalized Ising models, in Topol. Asp. Condens. Matter Phys. (Oxford University Press, 2017) pp. 219-240.

[68] S. Sanders and M. Holthaus, Hypergeometric continuation of divergent perturbation series: II. Comparison with Shanks transformation and Padé approximation, J. Phys. A Math. Theor. 
50, 465302 (2017).

[69] S. M. Chester, W. Landry, J. Liu, D. Poland, D. Simmons-Duffin, N. Su, and A. Vichi, Carving out OPE space and precise O(2) model critical exponents, J. High Energy Phys. 2020 (6), 142, arXiv:1912.03324.

[70] S. Rychkov, Conformal bootstrap and the $\lambda$-point specific heat experimental anomaly, J. Club Condens. Matter Phys. (2020).

[71] A. M. Shalaby, $\lambda$-point anomaly in view of the seven-loop hypergeometric resummation for the critical exponent $\nu$ of the $\mathrm{O}(2) \phi^{4}$ model, Phys. Rev. D 102, 105017 (2020). 